\title{
Erratum
}

\section{In Situ Generation of Palladium Nanoparticles: Reusable, Ligand-Free Heck Reaction in PEG-400 Assisted by Focused Microwave Irradiation}

Zhengyin Du,* Wanwei Zhou, Lin Bai,* Fen Wang, Jin-Xian Wang Synlett 2011, 369.

Reference 12 was cited incorrectly. The correct version is "V. Declerck, J. Martinez F. Lamaty Synlett 2006, 3029". 